\title{
THE REGULAR GRAPH OF A NONCOMMUTATIVE RING
}

\author{
S. AKBARI ${ }^{\otimes}$ and F. HEYDARI \\ (Received 29 November 2012; accepted 6 December 2012; first published online 13 February 2013)
}

\begin{abstract}
Let $R$ be a ring and $Z(R)$ be the set of all zero-divisors of $R$. The total graph of $R$, denoted by $T(\Gamma(R))$ is a graph with all elements of $R$ as vertices, and two distinct vertices $x, y \in R$ are adjacent if and only if $x+y \in Z(R)$. Let the regular graph of $R, \operatorname{Reg}(\Gamma(R))$, be the induced subgraph of $T(\Gamma(R))$ on the regular elements of $R$. In 2008, Anderson and Badawi proved that the girth of the total graph and the regular graph of a commutative ring are contained in the set $\{3,4, \infty\}$. In this paper, we extend this result to an arbitrary ring (not necessarily commutative). We also prove that if $R$ is a reduced left Noetherian ring and $2 \notin Z(R)$, then the chromatic number and the clique number of $\operatorname{Reg}(\Gamma(R))$ are the same and they are $2^{r}$, where $r$ is the number of minimal prime ideals of $R$. Among other results, we show that if $R$ is a semiprime left Noetherian ring and $\operatorname{Reg}(R)$ is finite, then $R$ is finite.
\end{abstract}

2010 Mathematics subject classification: primary 05C15; secondary 05C25, 16A.

Keywords and phrases: regular graph, total graph, girth, chromatic number.

\section{Introduction}

Throughout this paper we assume that $R$ is a ring (not necessarily commutative) with unity. Let $Z(R), Z_{l}(R)$ and $Z_{r}(R)$ be the set of zero-divisors, left zero-divisors and right zero-divisors of $R$, respectively. In fact, $Z(R)=Z_{l}(R) \cup Z_{r}(R)$. The total graph of $R, T(\Gamma(R))$, was first introduced in [4]. The vertex set of this graph consists of all elements of $R$, and for two distinct elements $x, y \in R$ the vertices $x$ and $y$ are adjacent if and only if $x+y \in Z(R)$. Let the regular graph of $R, \operatorname{Reg}(\Gamma(R))$, be the induced subgraph of $T(\Gamma(R))$ on the vertices of $\operatorname{Reg}(R)=R \backslash Z(R)$ and let $Z(\Gamma(R))$ be the induced subgraph of $T(\Gamma(R))$ on $Z(R)$. Clearly, the subgraph $Z(\Gamma(R))$ of $T(\Gamma(R))$ is connected, and $Z(\Gamma(R))$ is complete if and only if $Z(R)$ is a group under addition. Moreover, if $Z(R)$ is a group under addition, then no vertices of $Z(\Gamma(R))$ and $\operatorname{Reg}(\Gamma(R))$ are adjacent, and, as in [4, Theorem 2.2], one can easily prove that $\operatorname{Reg}(\Gamma(R))$ is a union of disjoint subgraphs, each of which is either a complete graph or a complete bipartite graph.

The Jacobson radical of $R$ and the group of units of $R$ are denoted by $J(R)$ and $U(R)$, respectively. We denote the characteristic of $R$ by char $R$. A ring $R$ is called reduced if

(c) 2013 Australian Mathematical Publishing Association Inc. 0004-9727/2013 \$16.00 
it has no nonzero nilpotent elements. The direct product of a family of rings $\left\{R_{i}: i \in I\right\}$ is denoted by $\prod_{i \in I} R_{i}$. As usual, $\mathbb{Z}_{n}$ denotes the integers modulo $n$. For any division ring $D, M_{n}(D)$ denotes the ring of all $n \times n$ matrices over $D$. Let $\mathrm{GL}_{n}(D)=U\left(M_{n}(D)\right)$. For any $i$ and $j, 1 \leq i, j \leq n$, we denote by $E_{i j}$ the matrix in $M_{n}(D)$ whose $(i, j)$ th entry is 1 and other entries are 0 .

Let $G$ be a graph. The girth of $G$, denoted by $\operatorname{gr}(G)$, is the length of a shortest cycle in $G(\operatorname{gr}(G)=\infty$ if $G$ contains no cycle). We denote the complete graph on $n$ vertices by $K_{n}$ and the complete bipartite graph with part sizes $m$ and $n$ by $K_{m, n}$. We call the complete bipartite graph $K_{1, n}$ a star. A graph $G$ is called regular if each vertex has the same number of neighbours. A clique in $G$ is a set of pairwise adjacent vertices and the clique number of $G$, denoted by $\omega(G)$, is the order of the largest clique in $G$. A $k$-vertex colouring of $G$ is an assignment of $k$ colours, $1, \ldots, k$, to the vertices of $G$. The $k$-vertex colouring is called proper if no two distinct adjacent vertices have the same colour. The smallest $k$ for which $G$ has the above property is called the chromatic number of $G$ and is denoted by $\chi(G)$. It is easy to see that $\chi(G) \geq \omega(G)$. A graph $G$ is called weakly perfect if $\chi(G)=\omega(G)$.

The investigation of graphs related to rings has been motivated by numerous applications. For example, let us mention that such graphs have been used in the study of applications of rings in coding theory (see the survey [3] and Sections 3.8, 3.14, 9.2 of the monograph [9]), automata theory (see [10]) and data mining (see [6, 12] and the broader bibliography in [11]). Many interesting results on graphs of rings have been obtained recently, for instance in $[1,16]$.

In [4], Anderson and Badawi proved that the girth of the total graph and the regular graph of a commutative ring are contained in the set $\{3,4, \infty\}$. In this paper, we extend this result to arbitrary rings (not necessarily commutative). Furthermore, we show that if $R$ is a finite ring, then $\operatorname{Reg}(\Gamma(R))$ is regular. In [2] it was proved that for every commutative ring $R$, if $R \backslash \bigcup_{i=1}^{n} I_{i}$ is finite, where $I_{1}, \ldots, I_{n}$ are proper ideals of $R$, then $R$ is finite. We also extend this result by showing that for an arbitrary ring $R$ and proper left ideals $I_{1}, \ldots, I_{n}$, if $R \backslash \bigcup_{i=1}^{n} I_{i}$ is finite, then $R$ is finite. As a corollary we show that for every reduced left Noetherian $\operatorname{ring} R$, if $\operatorname{Reg}(R)$ is finite, then $R$ is finite. We also generalise this result to semiprime left Noetherian rings. Among other results, we prove that for every reduced left Noetherian $\operatorname{ring} R$, if $2 \notin Z(R)$, then $\operatorname{Reg}(\Gamma(R))$ is weakly perfect. Moreover, we determine the exact value of $\chi(\operatorname{Reg}(\Gamma(R)))$.

\section{The girth of the total graph and the regular graph}

In this section we study the girth of the total graph and the regular graph of a ring $R$. Clearly, if there exist two nonzero distinct elements $x, y \in Z(R)$ such that $x+y \in Z(R)$, then $\operatorname{gr}(Z(\Gamma(R)))=3$; otherwise $Z(\Gamma(R))$ is a star with centre 0 . Therefore, $\operatorname{gr}(Z(\Gamma(R))) \in\{3, \infty\}$.

Theorem 2.1. Let $R$ be a ring. Then $\operatorname{gr}(\operatorname{Reg}(\Gamma(R))) \in\{3,4, \infty\}$.

Proof. First assume that $\operatorname{char} R=2$. We have two cases. 
Case 1. Suppose that $R$ contains a nonzero nilpotent element and $\operatorname{Reg}(\Gamma(R))$ contains a cycle $C$. Then $C$ contains two distinct vertices $x, y \in \operatorname{Reg}(R)$ such that $x, y \neq 1$ and $x+y \in Z(R)$. Let $0 \neq w \in R$ and $w^{2}=0$. If $w=w x=w y$, then $1+x$ and $1+y$ are zero-divisors of $R$, and thus $1-x-y-1$ is a 3-cycle in $\operatorname{Reg}(\Gamma(R))$. Now suppose that $w \neq w x$. (The case where $w \neq w y$ is similar.) If $w x w \notin\{0, w\}$ or $w x w=0$, then $1-(1+w)-(1+w x w)-1$ or $1-(1+w)-(1+w x)-1$ is a 3-cycle in $\operatorname{Reg}(\Gamma(R))$. Hence assume that $w x w=w$. So $x \neq 1+w$ and $w x \neq x w$. If $w x+x w \in Z(R)$, then $x-(x+w x)-(x+x w)-x$ is a triangle in $\operatorname{Reg}(\Gamma(R))$. Thus assume that $w x+x w \in \operatorname{Reg}(R)$. Now, if $x+w x+x w \in \operatorname{Reg}(R)$, then $x-(x+$ $w x)-(x+w x+x w)-(x+x w)-x$ is a 4-cycle in $\operatorname{Reg}(\Gamma(R))$. Therefore suppose that $x+w x+x w \in Z(R)$. We consider the following cases.

(i) $\quad w x+x w \neq 1,1+w$. Then $1-(1+w)-(w x+x w)-1$ is a 3-cycle in $\operatorname{Reg}(\Gamma(R))$, since $w(1+w+w x+x w)=w(1+w x+x w)=0$.

(ii) $w x+x w=1$. Then $x+1$ is a zero-divisor. If $x+1$ is a left zero-divisor, then $(x+w x)+(1+w)=(1+w)(x+1) \in Z(R)$. Also, since $x \neq 1, x+w x \neq 1+w$. Thus $x-1-(1+w)-(x+w x)-x$ is a 4-cycle in $\operatorname{Reg}(\Gamma(R))$. If $x+1$ is a right zero-divisor, then similarly $x-1-(1+w)-(x+x w)-x$ is a 4-cycle in $\operatorname{Reg}(\Gamma(R))$.

(iii) $w x+x w=1+w$. If $x+w \in \operatorname{Reg}(R)$, then $x-(1+w)-1-(x+w)-x$ is a 4-cycle in $\operatorname{Reg}(\Gamma(R))$. Thus assume that $x+w \in Z(R)$. If $x+1 \in Z(R)$, then $x-(1+w)-1-x$ is a triangle in $\operatorname{Reg}(\Gamma(R))$. Now suppose that $x+1 \in$ $\operatorname{Reg}(R)$. Then $x-(1+w)-(x+1)-(x+w x)-x$ is a 4-cycle in $\operatorname{Reg}(\Gamma(R))$. Note that $1+w \neq x+w x$, since $x \neq 1$. Also, $1+w x \in Z(R)$, since $(1+w x) w=0$.

Case 2. Suppose that $R$ is reduced and $\operatorname{Reg}(\Gamma(R))$ contains a cycle $C$. Then $C$ contains two distinct vertices $x, y \in \operatorname{Reg}(R)$ such that $x+y \in Z(R)$. If $x y=y x$, then $x^{2}+y^{2}=(x+y)^{2} \neq 0$. So $x^{2} \neq y^{2}$, and thus $x^{2}-x y-y^{2}-x^{2}$ is a triangle in $\operatorname{Reg}(\Gamma(R))$. Hence assume that $x y \neq y x$. Note that since $R$ is reduced, $Z(R)=Z_{l}(R)=$ $Z_{r}(R)$. Therefore if $x^{2} \neq y^{2}$, then $x^{2}-x y-y^{2}-y x-x^{2}$ is a 4-cycle in $\operatorname{Reg}(\Gamma(R))$. Also if $x^{2}=y^{2}$, then $x y+y x=(x+y)^{2} \in Z(R)$, and thus $x^{2}-x y-y x-x^{2}$ is a 3-cycle in $\operatorname{Reg}(\Gamma(R))$.

Next, assume that char $R \neq 2$ and $\operatorname{Reg}(\Gamma(R))$ contains a cycle $C$. Then $C$ contains two distinct vertices $x, y \in \operatorname{Reg}(R)$ such that $y \neq-x$ and $x+y \in Z(R)$. Thus $x-y-(-y)-(-x)-x$ is a 4-cycle in $\operatorname{Reg}(\Gamma(R))$.

From Theorem 2.1, we can deduce the next result in the case $\operatorname{gr}(\operatorname{Reg}(\Gamma(R)))=\infty$.

Corollary 2.2. Let $R$ be a ring. If $\operatorname{Reg}(\Gamma(R))$ is a forest, then $\operatorname{Reg}(\Gamma(R))$ is a disjoint union of some copies of $K_{1}$ or a disjoint union of some copies of $K_{2}$. In particular, if $\operatorname{Reg}(\Gamma(R))$ is a tree, then it has at most two vertices.

Proof. By the proof of Theorem 2.1, if char $R \neq 2$, then each $x \in \operatorname{Reg}(R)$ is only adjacent to $-x$. So $\operatorname{Reg}(\Gamma(R))$ is a disjoint union of some copies of $K_{2}$. Also, if char $R=2$ and $R$ is reduced, then no two distinct elements of $\operatorname{Reg}(R)$ are adjacent and thus $\operatorname{Reg}(\Gamma(R))$ is a disjoint union of some copies of $K_{1}$. (See the proof of 
Case 2 of Theorem 2.1.) Now suppose that char $R=2$ and $R$ contains an element $w \neq 0$ such that $w^{2}=0$. In view of the proof of Theorem 2.1 , we see that no two distinct elements of $\operatorname{Reg}(R) \backslash\{1\}$ are adjacent. We claim that $\operatorname{Reg}(R)=\{1,1+w\}$. Let $x \in \operatorname{Reg}(R) \backslash\{1,1+w\}$. Since $x$ and $x+x w$ are adjacent, $x+x w=1$ and $x+(1+w)=$ $x w+w \in Z(R)$. Therefore $x$ and $1+w$ are adjacent, a contradiction. So in this case $\operatorname{Reg}(\Gamma(R)) \cong K_{2}$.

Theorem 2.3. Let $R$ be a left Artinian ring and $\operatorname{Reg}(\Gamma(R))$ be a tree. Then $R$ is isomorphic to one of the rings

$$
\begin{aligned}
& \mathbb{Z}_{3}, \mathbb{Z}_{4}, \mathbb{Z}_{2}[x] /\left(x^{2}\right), \mathbb{Z}_{2}^{r}, \mathbb{Z}_{3} \times \mathbb{Z}_{2}^{r}, \mathbb{Z}_{4} \times \mathbb{Z}_{2}^{r}, \mathbb{Z}_{2}[x] /\left(x^{2}\right) \times \mathbb{Z}_{2}^{r}, \\
& \quad U T_{2}\left(\mathbb{Z}_{2}\right), U T_{2}\left(\mathbb{Z}_{2}\right) \times \mathbb{Z}_{2}^{r},
\end{aligned}
$$

where $U T_{2}\left(\mathbb{Z}_{2}\right)$ denotes the ring of $2 \times 2$ upper triangular matrices over $\mathbb{Z}_{2}$ and $r$ is a natural number.

Proof. By Corollary 2.2, $|U(R)| \leq 2$ and so by [19, Lemma 1.1], $R$ is finite. Clearly, $U(R)$ is cyclic. Therefore by [17, Theorem 1], if $R$ is an indecomposable ring (that is, not a direct product of two nontrivial rings), then $R$ is isomorphic to one of the rings

$$
\mathbb{Z}_{2}, \mathbb{Z}_{3}, \mathbb{Z}_{4}, \mathbb{Z}_{2}[x] /\left(x^{2}\right), U T_{2}\left(\mathbb{Z}_{2}\right)
$$

and otherwise $R$ is isomorphic to one of the rings

$$
\mathbb{Z}_{2}^{r}, \mathbb{Z}_{3} \times \mathbb{Z}_{2}^{r}, \mathbb{Z}_{4} \times \mathbb{Z}_{2}^{r}, \mathbb{Z}_{2}[x] /\left(x^{2}\right) \times \mathbb{Z}_{2}^{r}, U T_{2}\left(\mathbb{Z}_{2}\right) \times \mathbb{Z}_{2}^{r},
$$

where $r$ is a natural number.

We now determine the girth of the total graph of a ring.

Theorem 2.4. Let $R$ be a ring. Then $\operatorname{gr}(T(\Gamma(R))) \in\{3,4, \infty\}$.

Proof. Assume that $Z(R)$ is a group under addition. Then $Z(\Gamma(R))$ is a complete subgraph of $T(\Gamma(R))$ and no vertices of $Z(\Gamma(R))$ and $\operatorname{Reg}(\Gamma(R))$ are adjacent. So if $T(\Gamma(R))$ contains a cycle $C$, then all vertices of $C$ are contained in $Z(\Gamma(R))$ or $\operatorname{Reg}(\Gamma(R))$. Therefore by Theorem 2.1, $\operatorname{gr}(T(\Gamma(R))) \leq 4$.

Next, assume that there exist $x, y \in Z(R)$ such that $x+y \notin Z(R)$. If either $x \neq-x$ or $y \neq-y$, then either $0-x-(-x)-0$ or $0-y-(-y)-0$ is a triangle in $T(\Gamma(R))$. Thus suppose that $x=-x$ and $y=-y$. Then $0-x-(x+y)-y-0$ is a 4-cycle in $T(\Gamma(R))$. So $\operatorname{gr}(T(\Gamma(R))) \leq 4$.

THEOREM 2.5. Let $R$ be a ring and suppose that $Z(R)$ is not an additive group. If $Z(\Gamma(R))$ is a star, then $R \cong \mathbb{Z}_{2} \times \mathbb{Z}_{2}$ and $Z(\Gamma(R)) \cong K_{1,2}$.

Proof. Since $Z(R)$ is not closed under addition, we have $|Z(R)| \geq 3$. First, assume that $|Z(R)| \geq 4$ so there are two nonzero distinct elements $x, y \in R$ such that $x, y \in Z_{l}(R)$ or $x, y \in Z_{r}(R)$. With no loss of generality, assume that $x, y \in Z_{l}(R)$. Thus $R x \subseteq Z(R)$ and $R x=\{0, x\}$. Similarly, $R y=\{0, y\}$. Let $t=x+y$. Then $R t \subseteq\{0, x, y, t\}$. Since $Z(\Gamma(R))$ 
is a star, $t$ is regular and so $R \cong R / \operatorname{ann}_{l}(t) \cong R t\left(\right.$ where $\left.\operatorname{ann}_{l}(t)=\{a \in R: a t=0\}\right)$, a contradiction. Hence $|Z(R)|=3$ and thus, by [5, Theorem 1], $|R| \leq 9$. Clearly, $J(R)=0$. Now, by the Wedderburn-Artin theorem [13, Theorem 3.5], and noting that $|Z(R)|=3$, we conclude that $R \cong \mathbb{Z}_{2} \times \mathbb{Z}_{2}$. The last part is clear.

Remark 2.6. For a left Artinian ring $R, Z_{l}(R) \subseteq Z_{r}(R)$. To see this assume that $a, b \in R$ and $a b=0$, where $b \neq 0$. Then the left $R$-module homomorphism $T: R \rightarrow R$ with $T(x)=x a$, for $x \in R$, is not surjective and, by [14, p. 17], $T$ is not injective. Thus there is a nonzero element $x \in R$ such that $x a=0$.

THEOREM 2.7. Let $R$ be a finite ring. Then $\operatorname{Reg}(\Gamma(R))$ is regular.

Proof. Suppose that $t_{1}, \ldots, t_{k}$ are all neighbours of 1 in $\operatorname{Reg}(\Gamma(R))$, and $x \in \operatorname{Reg}(R)$. By Remark 2.6, it is clear that $x t_{1}, \ldots, x t_{k}$ are distinct vertices which are adjacent to $x$ in $\operatorname{Reg}(\Gamma(R))$. Now suppose that $y \in \operatorname{Reg}(R)$ is adjacent to $x$. Then $x+y \in Z(R)$ implies that $1+x^{-1} y \in Z(R)$. So there exists some $i, 1 \leq i \leq k$, such that $x^{-1} y=t_{i}$ and $y=x t_{i}$. Hence $x t_{1}, \ldots, x t_{k}$ are all neighbours of $x$. The proof is complete.

\section{The clique number and chromatic number of regular graphs}

In the next theorem we show that for every reduced left Noetherian ring $R$, if $2 \notin Z(R)$, then $\operatorname{Reg}(\Gamma(R))$ is weakly perfect. Moreover, we determine the exact value of $\chi(\operatorname{Reg}(\Gamma(R)))$. First we have the following remark.

Remark 3.1. Let $R$ be a left Noetherian ring and $P_{1}, \ldots, P_{r}$ be all minimal prime ideals of $R$ [7, Theorem 3.4]. If $R$ is reduced, then by [13, Lemma 12.6], $R / P_{i}$ is a domain, for $i=1, \ldots, r$. Thus by [7, Lemma 7.4], $Z(R)=\bigcup_{i=1}^{r} P_{i}$.

Theorem 3.2. Let $R$ be a left Noetherian ring and $2 \notin Z(R)$. If $R$ is reduced, then $\chi(\operatorname{Reg}(\Gamma(R)))=\omega(\operatorname{Reg}(\Gamma(R)))=2^{r}$, where $r$ is the number of minimal prime ideals of $R$.

Proof. By the previous remark, $Z(R)=\bigcup_{i=1}^{r} P_{i}$, where $P_{1}, \ldots, P_{r}$ are all distinct minimal prime ideals of $R$. Since $R / P_{i}$ is a domain, $\chi\left(\operatorname{Reg}\left(\Gamma\left(R / P_{i}\right)\right)\right)=2$, for $i=1, \ldots, r$. Now we assign to each $x \in \operatorname{Reg}(R)$, the colour $c(x)=\left(\delta_{1}, \ldots, \delta_{r}\right)$, where $\delta_{i}$ is the colour of $x+P_{i}$ in $\operatorname{Reg}\left(\Gamma\left(R / P_{i}\right)\right)$. We claim that this colouring is a proper colouring of $\operatorname{Reg}(\Gamma(R))$. Suppose that $x$ and $y$ are two distinct adjacent vertices of $\operatorname{Reg}(\Gamma(R))$. Thus there exists some $j$ such that $x+y \in P_{j}$. Since $2 \notin Z(R), x-y \notin P_{j}$. Hence $x+P_{j}$ and $y+P_{j}$ are two distinct adjacent vertices of $\operatorname{Reg}\left(\Gamma\left(R / P_{j}\right)\right)$. This implies that the colours of $x+P_{j}$ and $y+P_{j}$ are different in $\operatorname{Reg}\left(\Gamma\left(R / P_{j}\right)\right)$. Thus $c(x) \neq c(y)$ and so $\chi(\operatorname{Reg}(\Gamma(R))) \leq 2^{r}$.

Now we show that $\operatorname{Reg}(\Gamma(R))$ contains a clique of order $2^{r}$. If $r=1$, then clearly $\chi(\operatorname{Reg}(\Gamma(R)))=\omega(\operatorname{Reg}(\Gamma(R)))=2$. Thus assume that $r \geq 2$. Let $I_{j}=\prod_{i \neq j} P_{i}$ for $j=1, \ldots, r$. Note that $I_{j} \backslash P_{j}$ is nonempty, for $j=1, \ldots, r$. Thus there exists $x_{j} \in I_{j} \backslash P_{j}$ for each $j, 1 \leq j \leq r$. So $x_{j} \in P_{i}$, when $i \neq j$. We claim that the set $W=$ $\left\{t_{1} x_{1}+\cdots+t_{r} x_{r} \mid t_{j}= \pm 1\right.$ for $\left.j=1, \ldots, r\right\}$ forms a clique of order $2^{r}$ in $\operatorname{Reg}(\Gamma(R))$. 
First assume that $t_{1} x_{1}+\cdots+t_{r} x_{r}=t_{1}^{\prime} x_{1}+\cdots+t_{r}^{\prime} x_{r}$, where $t_{j}, t_{j}^{\prime}= \pm 1$. We show that $t_{j}^{\prime}=t_{j}$, for each $j, 1 \leq j \leq r$. With no loss of generality, suppose that there exists some $k, 1 \leq k \leq r$, such that $t_{j}^{\prime}=-t_{j}$ for $1 \leq j \leq k$ and $t_{j}^{\prime}=t_{j}$, for $k<j \leq r$. Then $2\left(t_{1} x_{1}+\cdots+t_{k} x_{k}\right)=0$ and, since $2 \notin Z(R), t_{1} x_{1}+\cdots+t_{k} x_{k}=0$. This implies that $x_{k} \in P_{k}$, a contradiction. So $|W|=2^{r}$ (note that $x_{j} \neq-x_{j}$ for $j=1, \ldots, r$ ). Next assume that $t_{1} x_{1}+\cdots+t_{r} x_{r} \in Z(R)$, where $t_{j}= \pm 1$, for $j=1, \ldots, r$. Thus there exists some $j$ such that $t_{1} x_{1}+\cdots+t_{r} x_{r} \in P_{j}$ which implies that $x_{j} \in P_{j}$, a contradiction. Therefore $W \subseteq \operatorname{Reg}(R)$. Finally, assume that $t_{1} x_{1}+\cdots+t_{r} x_{r} \neq t_{1}^{\prime} x_{1}+\cdots+t_{r}^{\prime} x_{r}$, where $t_{j}, t_{j}^{\prime}= \pm 1$. With no loss of generality, suppose that there exists some $k, 1 \leq k<r$, such that $t_{j}^{\prime}=t_{j}$ for $1 \leq j \leq k$ and $t_{j}^{\prime}=-t_{j}$ for $k<j \leq r$. Then $\left(t_{1} x_{1}+\cdots+t_{r} x_{r}\right)+\left(t_{1}^{\prime} x_{1}+\right.$ $\left.\cdots+t_{r}^{\prime} x_{r}\right)=2\left(t_{1} x_{1}+\cdots+t_{k} x_{k}\right) \in P_{k+1} \subseteq Z(R)$. Therefore the claim is proved. So $\chi(\operatorname{Reg}(\Gamma(R)))=\omega(\operatorname{Reg}(\Gamma(R)))=2^{r}$.

Corollary 3.3. Let $R$ be a left Artinian ring and $2 \notin Z(R)$. If $R$ is a reduced ring, then $\omega(\operatorname{Reg}(\Gamma(R)))=\chi(\operatorname{Reg}(\Gamma(R)))=2^{r}$, where $r$ is the number of maximal ideals of $R$.

We continue this section by the characterisation of rings whose regular graphs are complete.

Theorem 3.4. Let $R$ be a left Artinian ring. If $\operatorname{Reg}(\Gamma(R))$ contains a vertex adjacent to all other vertices, then $\operatorname{Reg}(\Gamma(R))$ is complete.

Proof. Suppose that $x \in \operatorname{Reg}(R)$ and $x$ is adjacent to all other vertices of $\operatorname{Reg}(\Gamma(R))$. Let $y, t \in \operatorname{Reg}(R)$ be two distinct vertices. We have $x \neq t y^{-1} x$, because if $x=t y^{-1} x$, then $\left(1-t y^{-1}\right) x=0$ which implies that $y=t$, a contradiction. Thus $x$ is adjacent to $t y^{-1} x$, that is, $x+t y^{-1} x \in Z(R)$. Hence by Remark 2.6, $y+t \in Z(R)$, that is, $y$ is adjacent to $t$. So $\operatorname{Reg}(\Gamma(R))$ is complete.

Lemma 3.5. Let $R$ be a simple left Artinian ring and $2 \notin Z(R)$. Then $\operatorname{Reg}(\Gamma(R))$ is a complete graph if and only if $R \cong \mathbb{Z}_{3}$.

Proof. By [13, Theorem 3.10], $R \cong M_{n}(D)$, where $D$ is a division ring and $n$ is a positive integer. If $n \geq 2$, then two matrices $I$ (the identity matrix) and $I+E_{n 1}$ are distinct elements of $\operatorname{Reg}(R)$ which are not adjacent, a contradiction. So $n=1$. If $|D|>3$, there exists $x \in D$ such that $x \neq 0,1,-1$. Thus $x$ is not adjacent to 1 , a contradiction. Hence $R \cong \mathbb{Z}_{3}$.

Lemma 3.6. Let $R=\prod_{i \in I} R_{i}$, where $R_{i}$ is a ring for each $i \in I$.

(1) If $\operatorname{Reg}\left(\Gamma\left(R_{i}\right)\right)$ is a complete graph and $2 \in Z\left(R_{i}\right)$, for some $i \in I$, then $\operatorname{Reg}(\Gamma(R))$ is a complete graph.

(2) If $\operatorname{Reg}(\Gamma(R))$ is a complete graph and $2 \notin Z(R)$, then $\operatorname{Reg}\left(\Gamma\left(R_{i}\right)\right)$ is a complete graph, for each $i \in I$.

Proof. Statement (1) follows from the fact that $\operatorname{Reg}\left(\prod_{i \in I} R_{i}\right)=\prod_{i \in I} \operatorname{Reg}\left(R_{i}\right)$. For (2), let $i \in I$ and for two distinct elements $x_{i}, y_{i} \in \operatorname{Reg}\left(R_{i}\right)$ consider $\left(1, \ldots, 1, x_{i}, 1,1, \ldots\right)$ and $\left(1, \ldots, 1, y_{i}, 1,1, \ldots\right)$. Now, since $\operatorname{Reg}(\Gamma(R))$ is complete and $2 \notin Z(R)$, we are done. 
TheOREM 3.7. Let $R$ be a ring and $2 \notin Z(R)$. If $\operatorname{Reg}(\Gamma(R))$ is a complete graph then $J(R)=0$.

Proof. Suppose that $u \in J(R)$ and $u \neq 0$. Since $2 \notin Z(R)$, we have $u \neq-u$. So $1+u$ and $1-u$ are two distinct elements of $\operatorname{Reg}(R)$ and since $\operatorname{Reg}(\Gamma(R))$ is a complete graph, $2=1+u+1-u \in Z(R)$, a contradiction.

Theorem 3.8. Let $R$ be a left Artinian ring and $2 \notin Z(R)$. Then $\operatorname{Reg}(\Gamma(R))$ is a complete graph if and only if $R \cong \mathbb{Z}_{3}^{r}$, for some natural number $r$.

Proof. By the previous theorem, $J(R)=0$, so, by the Wedderburn-Artin theorem, $R \cong M_{n_{1}}\left(D_{1}\right) \times \cdots \times M_{n_{r}}\left(D_{r}\right)$ for some division rings $D_{1}, \ldots, D_{r}$ and positive integers $n_{1}, \ldots, n_{r}, r$. By Lemmas 3.5 and 3.6, $R \cong \mathbb{Z}_{3}^{r}$. The converse is clear.

In [2, Theorem 2], it was proved that for every commutative $\operatorname{ring} R$, if $R \backslash \bigcup_{i=1}^{n} I_{i}$ is finite, then $R$ is finite, where $I_{i}$ is a proper ideal of $R$ for $i=1, \ldots, n$. We extend this result to one-sided ideals of an arbitrary ring.

THeOREM 3.9. Let $R$ be a ring and $I_{1}, \ldots, I_{n}$ be proper left ideals of $R$. If $R \backslash \bigcup_{i=1}^{n} I_{i}$ is finite, then $R$ is finite.

Proof. Clearly, we can assume that each $I_{i}$ is a maximal left ideal. We have the natural left $R$-module monomorphism $R / \bigcap_{i=1}^{n} I_{i} \rightarrow \prod_{i=1}^{n} R / I_{i}$. So $R / \bigcap_{i=1}^{n} I_{i}$ is Artinian (as a left $R$-module). On the other hand, $\bigcap_{i=1}^{n} I_{i}$ is finite, since $1+\bigcap_{i=1}^{n} I_{i} \subseteq R \backslash \bigcup_{i=1}^{n} I_{i}$. Thus $R$ is a left Artinian ring. Now, since $U(R)$ is finite, $R$ is finite [19, Lemma 1.1].

From the previous theorem, we have the following immediate consequence.

Corollary 3.10. Let $R$ be a reduced left Noetherian ring. If $\operatorname{Reg}(R)$ is finite, then $R$ is finite.

Proof. By Remark 3.1, $Z(R)$ is a finite union of prime ideals and so by the previous theorem the result holds.

By the Faith-Utumi theorem [15, p. 73], we can prove the following stronger result.

THeOREM 3.11. Let $R$ be a semiprime left Noetherian ring. If $\operatorname{Reg}(R)$ is finite, then $R$ is finite.

Proof. By Goldie's theorem [18, Theorem 3.2.14], suppose that $Q=M_{n_{1}}\left(D_{1}\right) \times \cdots \times$ $M_{n_{r}}\left(D_{r}\right)$ is the classical left quotient ring of $R$, where $D_{1}, \ldots, D_{r}$ are division rings and $n_{1}, \ldots, n_{r}, r$ are positive integers. By the Faith-Utumi theorem, $R$ contains $M_{n_{1}}\left(T_{1}\right) \times \cdots \times M_{n_{r}}\left(T_{r}\right)$, where $T_{i}$ is a domain (not necessarily with 1) whose classical left quotient ring is $D_{i}$, for $i=1, \ldots, r$. Since $\operatorname{Reg}(R)$ is finite, $T_{1}, \ldots, T_{r}$ are finite. Thus $D_{1}, \ldots, D_{r}$ are finite, and hence $R$ is finite.

We now propose the following conjecture.

Conjecture. Let $R$ be a left Noetherian ring. If $\operatorname{Reg}(R)$ is finite, then $R$ is finite. 
ReMArk 3.12. Let $D$ be a commutative Noetherian domain with the field of quotients $Q$. Let $R$ be a ring defined as follows:

$$
R=\left\{\left(\begin{array}{ll}
d & 0 \\
a & b
\end{array}\right) \mid d \in D, a, b \in Q\right\} .
$$

It is well known that this ring is left Noetherian, but it is not right Noetherian (see [8]). Obviously, if $d$ and $b$ are nonzero, then every element of $R$ of the form $\left(\begin{array}{ll}d & 0 \\ 0 & b\end{array}\right)$ is a regular element of $R$. If the number of regular elements of $R$ is finite, then $D$ and $Q$ should be finite and so $R$ is a finite ring. Thus the conjecture is true for $R$.

We close the paper with the following corollary.

Corollary 3.13. Let $R$ be a reduced left Noetherian ring and $2 \notin Z(R)$. If $\operatorname{Reg}(\Gamma(R))$ is complete, then $R \cong \mathbb{Z}_{3}^{r}$, for some natural number $r$.

Proof. By hypothesis, we have $\omega(\operatorname{Reg}(\Gamma(R)))=|\operatorname{Reg}(R)|$. Hence by Theorem 3.2, $\operatorname{Reg}(R)$ is finite and also by Corollary $3.10, R$ is finite. Now, by Theorem 3.8, the result holds.

\section{Acknowledgement}

The authors wish to express their appreciation to the referee for valuable suggestions and comments.

\section{References}

[1] M. Afkhami, M. Karimi and K. Khashyarmanesh, On the regular digraph of ideals of commutative rings, Bull. Aust. Math. Soc., to appear.

[2] S. Akbari, D. Kiani, F. Mohammadi and S Moradi, 'The total graph and regular graph of a commutative ring', J. Pure Appl. Algebra 213 (2009), 2224-2228.

[3] R. Alfaro and A. V. Kelarev, 'Recent results on ring constructions for error-correcting codes, algebraic structures and their representations', Contemp. Math. 376 (2005), 1-12.

[4] D. F. Anderson and A. Badawi, 'The total graph of a commutative ring', J. Algebra 320 (2008), 2706-2719.

[5] N. Ganesan, 'Properties of rings with a finite number of zero-divisors II', Math. Ann. 161 (1965), 241-246.

[6] D. Y. Gao, A. V. Kelarev and J. L. Yearwood, 'Optimization of matrix semirings for classification systems’, Bull. Aust. Math. Soc. 84 (2011), 492-503.

[7] K. R. Goodearl and R. B. Warfield Jr, An Introduction to Noncommutative Noetherian Rings (Cambridge University Press, Cambridge, 2004).

[8] I. N. Herstein, 'A counterexample in Noetherian rings', Proc. Natl. Acad. Sci. USA. 54 (1965), 1036-1037.

[9] A. V. Kelarev, Ring Constructions and Applications (World Scientific, River Edge, NJ, 2002).

[10] A. V. Kelarev and D. S. Passman, 'A description of incidence rings of group automata', Contemp. Math. 456 (2008), 27-33.

[11] A. Kelarev, J. Ryan and J. Yearwood, 'Cayley graphs as classifiers for data mining: the influence of asymmetries', Discrete Math. 309(17) (2009), 5360-5369.

[12] A. V. Kelarev, J. L. Yearwood and P. W. Vamplew, 'A polynomial ring construction for classification of data', Bull. Aust. Math. Soc. 79 (2009), 213-225. 
[13] T. Y. Lam, A First Course in Noncommutative Rings (Springer, Berlin, 1991).

[14] T. Y. Lam, Lectures on Modules and Rings (Springer, Berlin, 1998).

[15] J. C. McConnell and J. C. Robson, Noncommutative Noetherian Rings, revised edition, Graduate Studies in Mathematics, 30 (American Mathematical Society, Providence, RI, 2001).

[16] S. M. Moconja and Z. Z. Petrović, 'On the structure of comaximal graphs of commutative rings with identity', Bull. Aust. Math. Soc. 83(1) (2011), 11-21.

[17] R. Raghavendran, 'A class of finite rings', Compositio Math. 22 (1970), 49-57.

[18] L. H. Rowen, Ring Theory, Vol. I (Academic Press, Boston, 1988).

[19] I. Stewart, 'Finite rings with a specified group of units', Math. Z. 126 (1972), 51-58.

S. AKBARI, Department of Mathematical Sciences, Sharif University of Technology, Tehran, Iran

e-mail: s_akbari@sharif.edu

F. HEYDARI, Department of Mathematics, Karaj Branch, Islamic Azad University, Karaj, Iran

e-mail: f-heydari@kiau.ac.ir 